\title{
Analyzing the Critical Path for the Well-Formed Workflow Schema*
}

\author{
Jin Hyun Son and Myoung Ho Kim \\ Dept. of Electrical Engineering and Computer Science, KAIST, \\ 373-1 Kusong-dong Yusong-gu Taejon 305-701 Korea \\ \{jhson, mhkim\}@ dbserver.kaist.ac.kr
}

\begin{abstract}
Though the concept of the critical path in the workflow is important because it can be utilized in many issues in workflow systems, the critical path in the context of the workflow has not been much addressed in the past. In this paper we propose a method to systematically determine the critical path in a workflow schema built by the workflow control constructs described in our workflow model.
\end{abstract}

\section{Introduction}

Nowadays, the concept of a workflow attracts a great interest in an objective of automating and computerizing business processes in whole or part. A business process is abstracted to a workflow which is composed of "a collection of activities" interconnected by various workflow control flows [1]. The critical path in a workflow schema is defined as "the longest execution path in a workflow schema" which directly affects the total workflow completion time. The activities in the critical path are called critical activities. We expect that the concept of the critical path and the critical activity can be effectively utilized in many workflow issues, for example, workflow resource/time management.

In Section 2, we describe the workflow control constructs and model considered in the paper. Section 3 presents our proposed method to determine the critical path in a workflow. Finally, we summary the paper in Section 4.

\section{Workflow Control Constructs and Model}

A workflow schema, which is a description of a workflow, is a network of nodes and directed edges. It begins from the start node and ends with the final node. Each node denotes an activity, and each directed edge denotes a transition between two nodes, i.e., a branch of execution. Figure

\footnotetext{
${ }^{*}$ This work was supported in part by the Hacking and Virus research Center
}

1 shows graphical expressions of various workflow control constructs with which we can fairly describe current complicated business logic. Note that for a split control construct $\alpha$, there is a set of join control construct $C_{\alpha}$ that can be combined with $\alpha$ whose resulting workflow schema can produce a semantically meaningful flow of control. For example, $C_{A N D-\text { split }}=\{A N D-j o i n$, Selective $-A N D-$ join, Preference $-O R-j$ oin $\}$.

Definition 1 Any subnetwork in a workflow schema that satisfies one of the following conditions is called a nonsequential control block(, simply control block). 1) A subnetwork that starts from a split control construct and ends with a matching join control construct. 2) A subnetwork that consists of all the nodes and edges between the source and the destination of the LOOP.

Definition 2 Non-sequential control blocks and their nested control blocks are called well-formed control blocks. A workflow schema composed of well-formed control blocks and simple nodes is called a well-formed workflow schema.

When we assume that workflow requests arrive by a Poisson process and the service time in each activity is exponential, a well-formed workflow schema can be modeled

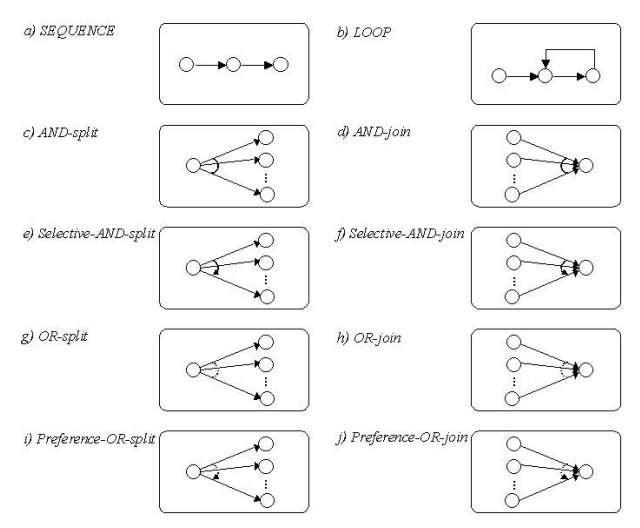

Figure 1. Workflow Control Constructs 


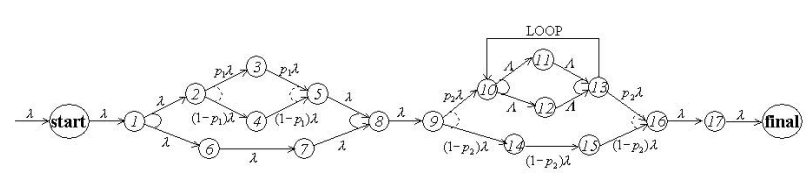

Figure 2. Workflow Queuing Network

as a $\mathrm{M} / \mathrm{M} / 1$ queuing network such as telephone networks or computer communication networks, which allows us to analyze some important workflow properties. In a $\mathrm{M} / \mathrm{M} / 1$ workflow queuing network, each activity is an independent $\mathrm{M} / \mathrm{M} / 1$ queuing system [2]. We can, therefore, specify the arrival and departure rate in each activity as in Figure 2 from the information of the initial request rate, the service rate in each activity, and the branch probabilities in OR-split, Preference-OR-split, and LOOP control constructs.

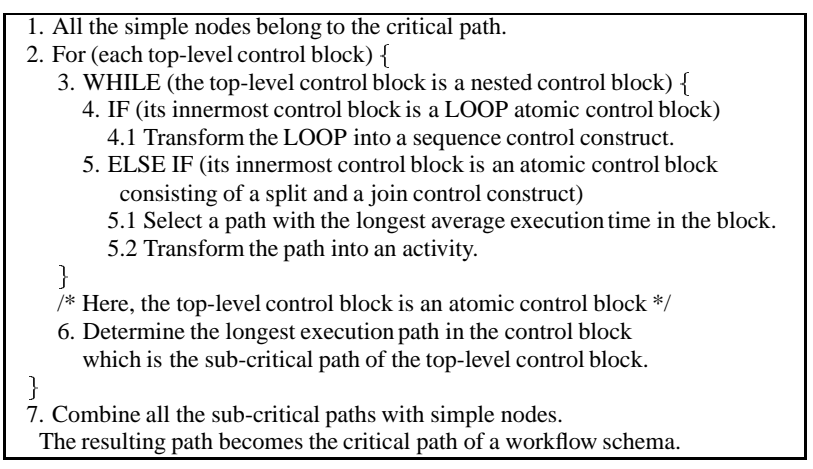

Figure 3. CPWS Algorithm

\section{Finding the Critical Path}

The critical path in a workflow schema is the longest execution path from the start node to the final node. From the aforementioned workflow queuing network, we propose a method called the Critical Path in a Workflow Schema (CPWS) that determines the critical path in a workflow schema. As many workflow instances for the same workflow schema can be concurrently executed, some workflow instances should wait at the queue of an activity during other workflow instances that arrives ahead are served. This means that the average execution time of a workflow instance in an activity is the average service time in the activity plus the average waiting time at the queue of the activity.

It is clear that since a workflow schema is a sequence of simple nodes and/or top-level control blocks, all the simple nodes and the longest execution path in a top-level control block are to be part of the critical path of a workflow schema. Therefore, CPWS basically finds out a sub-critical path with the longest average execution time in each toplevel control block and then combines these sub-critical

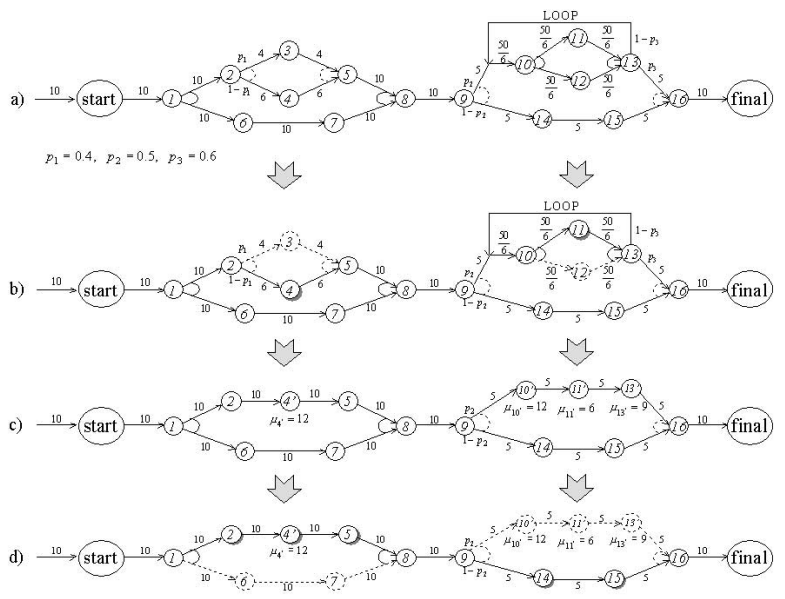

Figure 4. An Overall Example of the CPWS

paths to form the critical path as explained in Figure 3. Finding a subcritical path in each control block, which corresponds to the step 4, step 5, and step 6 in the CPWS algorithm, can be easily performed using the queuing theory.

Figure 4 show the overall example of our CPWS method. Let the service rate to the workflow schema be 10 and the branch probabilities $p_{1}, p_{2}$, and $p_{3}$ be $0.4,0.5$, and 0.6 , respectively. Thus, we can specify the arrival and departure rate at each activity as in Figure 4-(a), which is based on Section 2 . And, let the service rate $\mu_{i}$ of activity $i$ be $\left[\mu_{1}, \mu_{2}, \cdots, \mu_{15}\right]=$ $[13,15,7,8,20,18,20,14,16,20,10,15,15,6,7,25]$. As a result, we can determine the critical path, $\{1,2,4,5,8,9$, $14,15,16\}$, in the workflow schema by combining these sub-critical paths.

\section{Conclusion}

We understand that the concept of the critical path may be very useful because it can be utilized in many workflow issues, for example, workflow resource management, workflow time management, etc. In this paper we first specify various workflow control constructs which can be enough to support the context of current complex business processes. And then, we propose a method to determine the critical path in a well-formed workflow schema.

\section{References}

[1] P. Lawrence. Workflow Handbook 1997. John Wiley \& Sons Ltd, 1997.

[2] R. W. Wolff. Stochastic Modeling and the Theory of Queues. Prentice Hall, 1989. 